\title{
Polysulphone composite membranes modified with two types of carbon additives as a potential material for bone tissue regeneration
}

\author{
ALICJA WEDEL-GRZENDA ${ }^{1}$, ANETA FRACZEK-SZCZYPTA ${ }^{1, *}$, MAURICIO TERRONES ${ }^{2,3}$, \\ ANA LAURA ELÍAS ${ }^{2}$, MALGORZATA LEKKA ${ }^{4}$, ELZBIETA MENASZEK ${ }^{5}$ \\ and STANISLAW BLAZEWICZ ${ }^{1}$ \\ ${ }^{1}$ AGH-University of Science and Technology, Faculty of Materials Science and Ceramics, Department of Biomaterials, \\ Al. Mickiewicza 30, 30-059 Krakow, Poland \\ ${ }^{2}$ The Pennsylvania State University, Department of Physics and Center for 2-Dimensional and Layered Materials, \\ 104 Davey Laboratory, University Park, PA 16802, USA \\ ${ }^{3}$ Shinshu University, Research Center for Exotic Nanocarbons (JST), Wakasato 4-17-1, Nagano-city 380-8553, Japan \\ ${ }^{4}$ The Henryk Niewodniczanski Institute of Nuclear Physics, Polish Academy of Sciences; Department of Experimental \\ Physics of Complex Systems, Laboratory of Biophysical Microstructures, Radzikowskiego 52, 31-342 Krakow, Poland \\ ${ }^{5}$ Collegium Medicum, Jagiellonian University, Faculty of Pharmacy, Department of Cytobiology, Medyczna 9, 30-068 \\ Krakow, Poland
}

MS received 26 May 2016; accepted 24 June 2016

\begin{abstract}
This study presents a detailed evaluation of the impact of carbon fibrous materials on the physicochemical properties of polysulphone (PSU) membranes and their preliminary osteoblast-like cells response in vitro. Multiwalled carbon nanotubes (MWCNTs) and short carbon fibres (SCFs) were incorporated into PSU and membranes were produced by the phase inversion method. Then, the physicochemical properties of the membranes' surface were investigated. Scanning electron microscopy (SEM) was used to evaluate microstructure and porosity. Surface properties such as roughness, wettability and surface energy were evaluated using atomic force microscopy (AFM), contact profilometry and a goniometer, respectively. The presence of carbon fibrous additives in the PSU matrix improved its hydrophilicity. Porosity and topography of the PSU membranes were also changed upon incorporation of carbon additives. The mechanical properties of the PSU membranes were improved after SCF addition. All physicochemical properties of the obtained composite membranes had significant impact on the osteoblast-like cells response. Preliminary viability tests indicated biocompatibility of all membranes.
\end{abstract}

Keywords. Carbon additives; polysulphone; composite membranes; surface properties; mechanical properties; MG-63 cells viability.

\section{Introduction}

One of the interesting areas of polymer application in biomedicine is the fabrication of membranes. Membranes produced for medical purposes require similar production steps like those produced for industrial purposes; however, they have a much higher financial value [1]. In biomedicine, membranes are applied in artificial organs, such as artificial livers, artificial kidneys or pancreas systems. They are also used in drug delivery systems [2]. Another flourishing field of membrane application is tissue engineering [3].

The idea of creating a space in which healing is promoted is not a new one. This concept was introduced for membranes about half a century ago, when researchers used cellulose acetate filters in nerve regeneration. Other experiments revealed the formation of new bone tissue under polymer cages placed in dogs' femoral defects [4]. The principle of guided tissue regeneration (GTR) was first introduced in the 1980s. Then, on the basis of these principles, a more detailed

*Author for correspondence (afraczek@agh.edu.pl) technique — guided bone regeneration (GBR) — was developed. Since membranes act as barriers, they can assist in the bone regeneration process in GBR, protecting the bone defect from soft tissue invasion [4-6].

Non-resorbable as well as resorbable membranes are used in GBR [7]. One of the non-resorbable polymer with the potential to be used as membrane material for GBR is polysulphone (PSU). The reactivity of PSU opens the possibility for its functionalization and modification [8]. One of the approaches to improve the properties of pure PSU is its modification using different types of additives in the micro- and nanoscale, which results in obtaining micro- and nanocomposite membranes. Among the many types of additives in the micro- and nanoscale, carbon fibres and carbon nanotubes (CNTs) are promising candidates for modification of polymer membranes for biomedical applications.

Carbon fibres have been applied as bone substitutes and as a reinforcement phase of polymer composites for a long time. In recent approaches, carbon fibres were modified with ceramic particles to improve their bioactivity [9-12]. It is important to note that the biocompatibility of the carbon 
fibres depends on their structure, crystallinity, morphology and surface chemistry [9].

Nanotechnology provides completely new possibilities, and carbon-based nanotechnology has been developing rapidly since the discovery of CNTs by Iijima in 1991 [13]. These cylinder-shaped nanotubes made of $\mathrm{sp}^{2}$ bonded carbon atoms have extraordinary mechanical and electrical properties, which make them suitable particularly for biomedical applications [14].

Opinions on the toxicity of carbon nanomaterials, especially CNTs, are divided. However, many researchers have shown that there is substantial evidence of their biocompatibility in contact with, e.g., bone cells [15-20]. Applications of CNTs as modifiers in polymer composites for regeneration or reconstruction of bone are popular [15,18-20]. In a biostable polymer such as PSU, CNTs influence the physicochemical properties and morphology [21]. Researchers have found an improvement in the hydrophilicity of composite membranes. Also, the addition of up to $1.5 \mathrm{wt} \%$ MWCNTs increased the pore size of membranes, and an addition of $4 \mathrm{wt} \%$ multi-walled carbon nanotubes (MWCNTs) into PSU increased rejection and flux of membranes [21]. Stankova et al [20] fabricated PSU composites with both MWCNTs and singlewalled carbon nanohorns (SWCNHs). Research revealed a higher Young's modulus and increased tensile strength values of these materials when compared with pure PSU samples. Moreover, these nanocomposites improved adhesion and viability of MG-63 osteoblast-like cells.

CNTs and fibres are interesting materials with the ability to modify polymer properties for biological applications. In this work, we compared two composite membranes based on PSU modified with two types of fibrous carbon materials obtained by phase inversion. This method is very popular to obtain PSU membranes for various applications, e.g., in haemodialysis, water and gas purification, etc. [8,22,23]. However, the nature of the solvent used to dissolve the polymer, type of anti-solvent and the process conditions of the phase inversion have a significant effect on the properties of the membrane and could be changed in a large range [24,25].

Despite a similar chemical composition, these carbon additives have significantly different properties and dimensions. While information on the preparation of PSU membranes with CNTs using phase inversion exists in the literature $[8,24,26,27]$, the information on the membranes reinforced with short carbon fibres is very limited [28], especially in reference to biological application. The main purpose was to verify whether and how the existence of two phases of carbon additives with different properties affects the physicochemical, mechanical and preliminary biological properties of the composite membranes.

\section{Materials and methods}

\subsection{Investigated materials}

- PSU (Aldrich Chemical Company Inc.) in the form of pellets $\left(M_{\mathrm{n}} \sim 26.000\right)$ was used in the studies. The choice of PSU was due to its good mechanical properties, high chemical resistance, the ability to form membranes and especially because of its proven biocompatibility.

- Low Young's modulus carbon fibres (Soficar) in the shape of long yarns were sectioned and then milled.

- MWCNTs were produced by chemical vapour deposition (CVD) described elsewhere [29], using toluene $\left(\mathrm{C}_{7} \mathrm{H}_{8}\right)$ as a carbon source and ferrocene $\left(\mathrm{FeCp}_{2}\right)$ as a catalyst precursor. As-grown samples were nitrided and oxygenated. The MWCNTs had a mean diameter of $65 \mathrm{~nm}$ and a length of $1.4 \mu \mathrm{m}$.

PSU membranes and composite membranes were fabricated by phase inversion. Dimethylformamide (DMF) (POCH SA) was used as a solvent for the polymer. Pure polymeric membranes were initially fabricated as control samples. The concentration of PSU was $15 \%$. Additionally, a solution of polyvinylpirrolidone (PVP, Sigma-Aldrich) in DMF was added to the PSU solution. PVP is commonly used in membrane production and allows controlling pore size and distribution in the material; it also increases permeability and hydrophilicity and reduces fouling [30]. The proportion by volume of PSU/DMF to PVP/DMF was 3:1. The solution was homogenized with a magnetic stirrer for $15 \mathrm{~min}$.

Figure 1 shows a diagram of the preparation of composite membranes. The appropriate amount of carbon additive (in reference to the dry PSU mass) was mixed with the solvent and sonicated using an ultrasonic tip for 3 min to obtain good dispersion and homogenization. After dispersion of carbon additives in DMF, PSU was added and the solution was mixed for $24 \mathrm{~h}$ on a magnetic stirrer. The proposed process of CNTs homogenization in the polymer, preceded by dispersion in solvent, results from previous experience of authors in the preparation of nanocomposites based on these nanoadditives [31,32]. A similar procedure for the preparation of nanotubes-PSU solutions has been proposed in other publications [24]. The suggested method of the preparation of solutions and the application of phase inversion method for obtaining membranes with the short carbon fibres (SCFs) have not been published so far.

Membranes were fabricated with the use of a doctor blade with water as the coagulation bath. The thickness of the produced membranes was $550 \mu \mathrm{m}$. Membranes were rinsed several times in water to remove the solvent and dried for 3 days at room temperature. For further studies, the following three materials were chosen:

- PSU - pure PSU membrane (15\% PSU in DMF), used as a reference material;

- $\mathrm{PSU}+2 \% \mathrm{SCF}$ - PSU membrane (15\% PSU in DMF) modified with 2 wt $\%$ SCFs;

- $\mathrm{PSU}+2 \% \mathrm{CNT}$ - PSU membrane (15\% PSU in DMF) modified with $2 \mathrm{wt} \%$ functionalized MWCNTs.

\subsection{Methods of samples characterization}

Microstructure and morphology of SCFs, MWCNTs and the obtained membranes were examined using scanning electron 


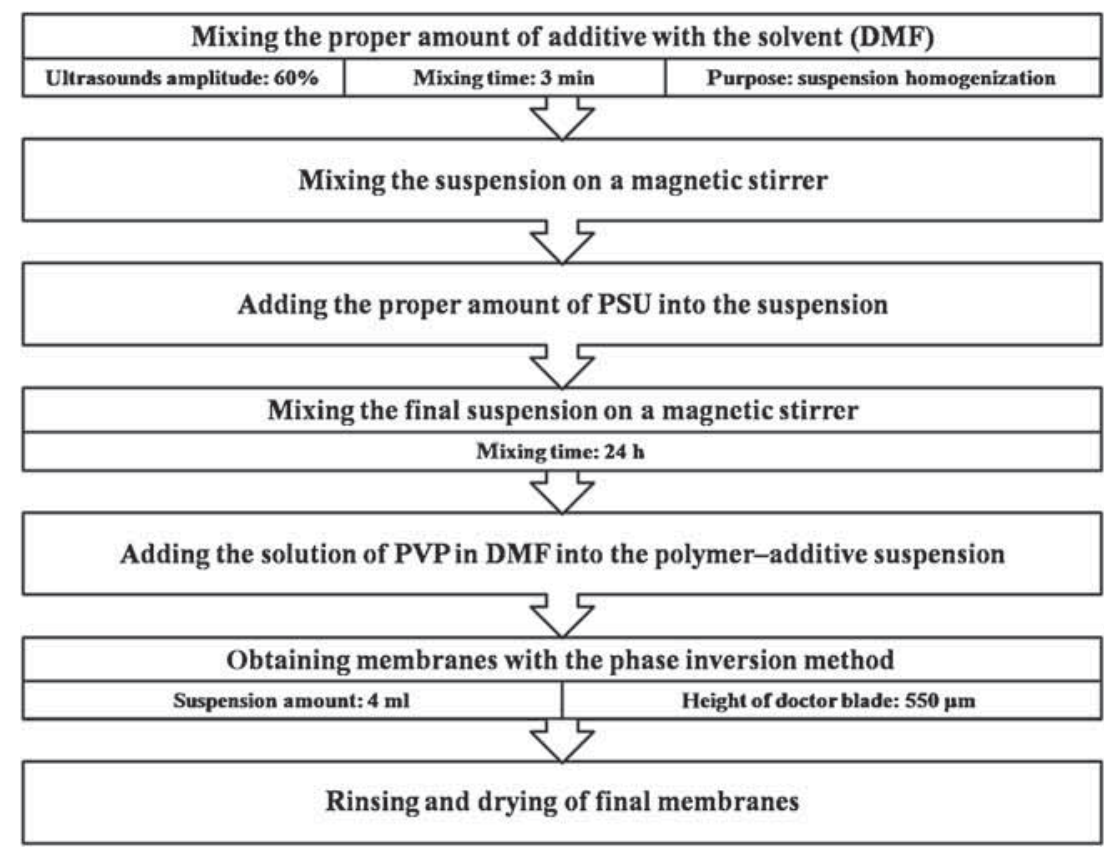

Figure 1. The process of composite membranes preparation.

microscopy (SEM, Nova NanoSEM 200, FEI). The porosity of the samples was analysed on the basis of SEM microphotographs using the ImageJ software. For each material, the diameters of several dozen pores were measured from images with a magnification of $350 \times$. Additionally, the topography of the PSU+2\%SCF membrane was examined using an inverted microscope with phase contrast (Axiovert 40, Zeiss) to observe the alignment of SCFs in the polymer.

Measurements of surface morphology were carried out using the profilometry technique (Hommel Tester T1500 and atomic force microscopy (AFM XE120, Park Systems, South Korea). The surface was imaged with a triangular, silicon nitride cantilever MLCT-D (Bruker AFM Probes) with a spring constant of $0.03 \mathrm{~N} \mathrm{~m}^{-1}$ and a nominal resonant frequency of $15 \mathrm{kHz}$. Measurements were performed in contact mode, with a scanning rate of $1.5 \mathrm{~Hz}$. Maps of randomly chosen regions with scan size of $60 \mu \mathrm{m} \times 60 \mu \mathrm{m}$, i.e., $256 \times 256$ pixels, were chosen. Six images were recorded for each sample type, which were analysed using the XE Image Processing Program. Surface morphology was quantified using the following parameters: average roughness $\left(R_{\mathrm{a}}\right)$ and tenpoint average roughnesses $\left(R_{\mathrm{Z}}\right)$, determined for four maps recorded for each studied sample type.

Contact angle was measured by the sessile drop method using the automatic drop shape analysis system DSA 10Mk2 (Kruss, Germany). UHQ-water (produced by Purelab UHQ, Elga, Germany) drops of a volume of $0.2 \mu \mathrm{l}$ were put onto each sample and the contact angle was calculated by averaging the results of 10 measurements. A pure PSU membrane was used as a reference sample.

Surface energy of membranes was determined using the Owens-Wendt method [33]. This procedure involves measuring the contact angle of the two liquids of known surface tensions (water and diiodomethane) on the samples. Values of polar and dispersive energies for water were $\gamma_{p}=$ $51 \mathrm{~mJ} \mathrm{~m}^{-2}$ and $\gamma_{\mathrm{d}}=21.8 \mathrm{~mJ} \mathrm{~m}^{-2}$, respectively, and for diiodomethane $\gamma_{\mathrm{p}}=2.3 \mathrm{~mJ} \mathrm{~m}^{-2}$ and $\gamma_{\mathrm{d}}=48.5 \mathrm{~mJ} \mathrm{~m}^{-2}$, respectively.

Tensile mechanical tests of the membranes modified with SCFs and MWCNTs were performed using samples with a fixed dimension of $5 \times 40 \times 0.1 \mathrm{~mm}^{3}$. Tensile strength and Young's modulus were determined with a universal testing machine (Zwick, model 1435) controlled by the TestXpert v.8.1 software, with a crosshead speed of $5 \mathrm{~mm} \mathrm{~min}^{-1}$. Tensile modulus was evaluated from the slope of the initial, linear part of the force-strain function. The quantitative results were presented as mean \pm standard error of mean (SEM), calculated from six individual measurements. Measuring changes in the mechanical properties of the membranes is a particularly useful method for the evaluation of the effectiveness of the dispersion process.

Preliminary in vitro test was performed using human osteoblast-like MG-63 cells (ATCC, UK). The cells were cultured in Eagle Minimum Essential Medium (ATCC, UK) supplemented with $10 \%$ inactivated fetal bovine serum (HyClone, Germany) at $37^{\circ} \mathrm{C}$ in a humidified air atmosphere containing $5 \%$ of $\mathrm{CO}_{2}$.

For in vitro tests, membranes were immersed in $70 \%$ ethanol and sterilized with UV radiation for $0.5 \mathrm{~h}$ on each side. Sterile disks cut out from membranes were placed into 24-well culture plates and MG-63 cells were seeded on their surface at a density of $2 \times 10^{5} \mathrm{ml}^{-1}$ per well. The cells were cultured in contact with the membranes for 3 and 7 days.

To determine cell viability, a CellTiter $96^{\circledR}$ AQueous One Solution Cell Proliferation Assay (Promega GmbH, Germany) was used. This reagent is responsible for the 
bioreduction of a tetrazolium salt to a coloured product (formazan), which is soluble in the culture medium. The quantity of formazan, which is proportional to the number of living cells, was measured spectrophotometrically in the POLARStar Omega (BMG LABTECH, Germany) reader. Results were expressed as mean \pm SD obtained from 5-8 samples for each experimental group. Significant effects $(p<0.05)$ were determined using the unpaired Student's $t$-test.

\section{Results and discussion}

\subsection{Microstructure and topography}

The morphology of SCFs and MWCNTs is presented in figure $2 a$ and $b$, respectively. The morphology of pure PSU membranes indicated the presence of a bimodal pore distribution in the membrane. Pores in the micrometre size range and below could be observed in the material (figure $3 a$ and $b$ ), where smaller pores were placed between bigger pores and around their edges. Figure $3 \mathrm{c}$ shows microphotographs of the membrane's cross-section. Parallel, tubular pores that allow ion transport from one side of the membrane to another are observed. PSU membranes have a hierarchical structure that mimics the structure of natural bones and results in a better match of material applied in GTR; it also allows the transport of both nutrients and waste products to the interior and exterior of the tissue.

Morphology of PSU+2\%SCF membrane is presented in figure $4 \mathrm{a}$ and $\mathrm{b}$. Similar to the pure PSU membrane, a lot of micrometric spherical pores (figure 4a) can be observed; however, for PSU+2\%SCF, some of these pores were partly closed compared with the pure PSU membrane. Smaller pores (below micrometres), shown in figure $4 \mathrm{~b}$, were also present between bigger pores and around their edges. A cross-section of a PSU $+2 \%$ SCF membrane revealed a network of parallel pores in which the carbon fibres can be seen (figure 4c, arrows).

Microphotographs shown in figure $5 \mathrm{a}-\mathrm{c}$ exhibit the microstructure of the PSU $+2 \% \mathrm{CNT}$ membrane. Porosity was similar to that observed for PSU and PSU $+2 \% \mathrm{SCF}$
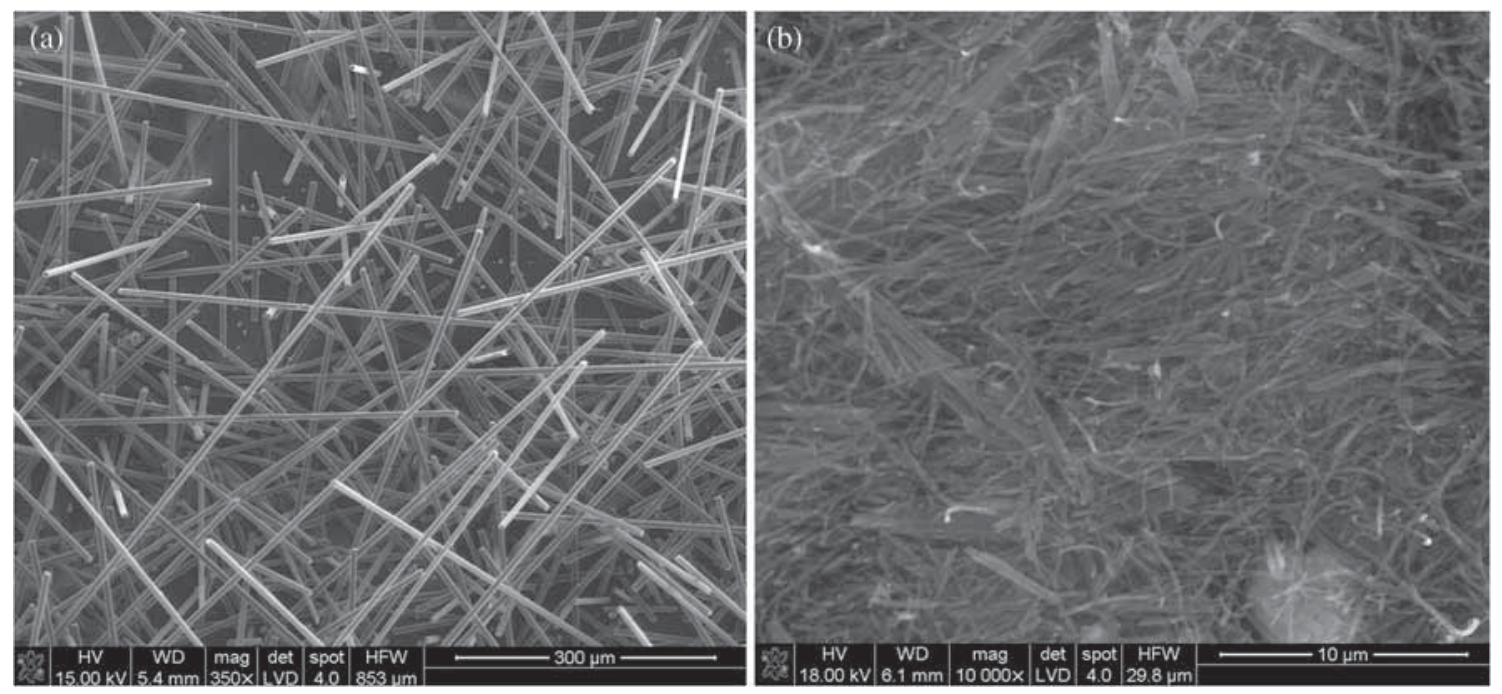

Figure 2. SEM microphotographs of (a) SCF, magnification $600 \times$ and (b) CNT, magnification $10000 \times$.
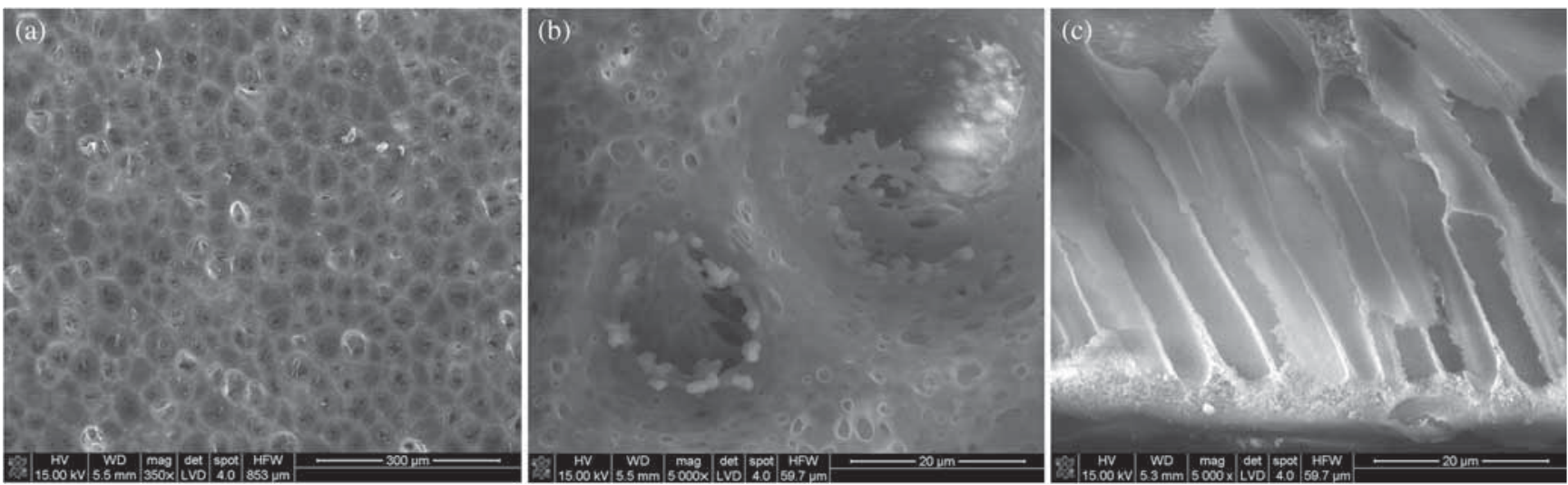

Figure 3. SEM microphotographs of PSU membrane surface: (a) magnification $350 \times$ and (b) magnification $5000 \times$ and $(\mathbf{c})$ cross-section of PSU membrane, magnification 5000×. 

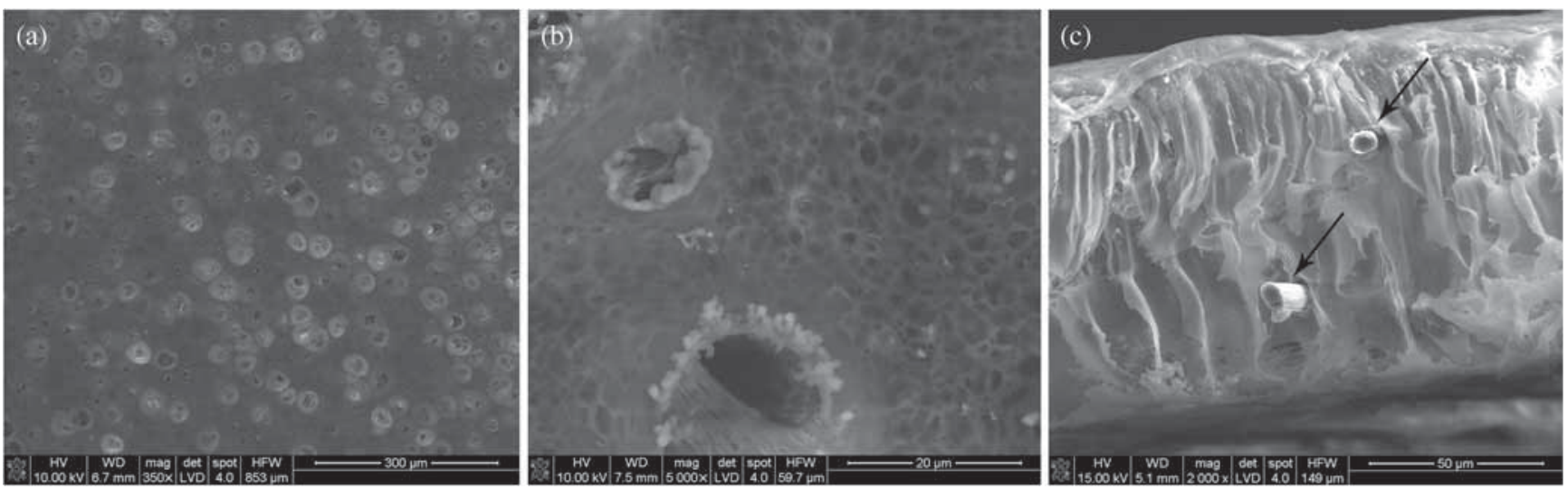

Figure 4. SEM microphotographs of PSU $+2 \%$ SCF membrane surface: (a) magnification $350 \times$ and (b) magnification $5000 \times$ and (c) cross-section of PSU+2\% SCF membrane, magnification $2000 \times$.
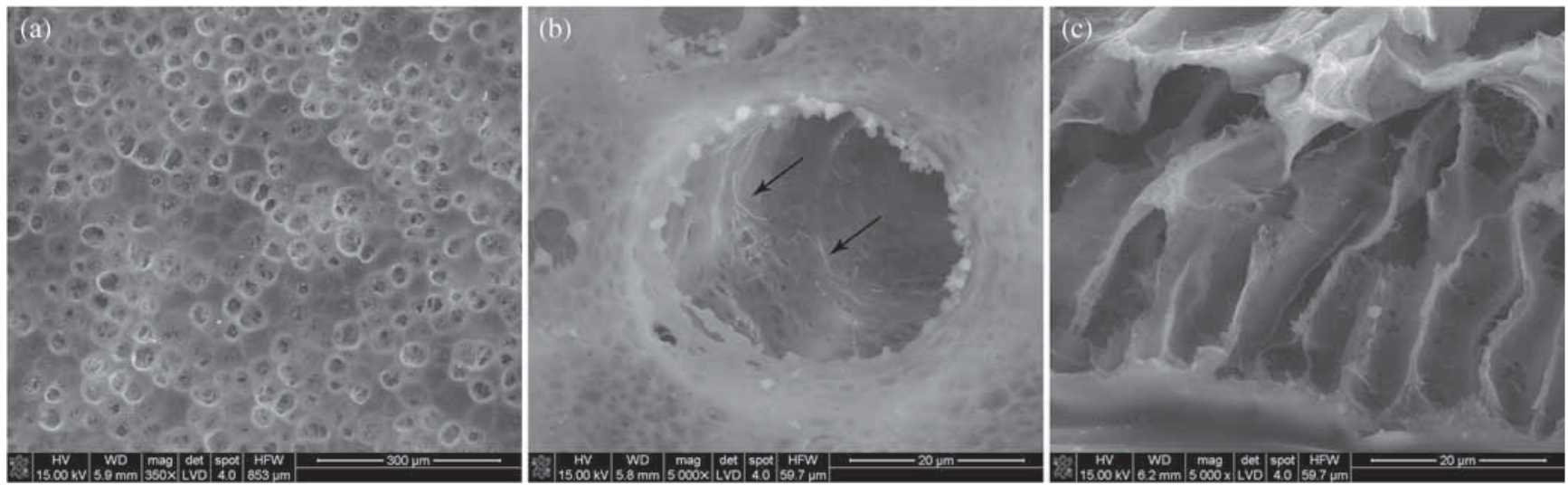

Figure 5. SEM microphotographs of PSU+2\%CNT membrane surface: (a) magnification $350 \times$, (b) magnification $5000 \times$ and (c) cross-section of PSU+2\%CNT membrane, magnification $5000 \times$.

membranes, but the fraction of bigger pores was greater in comparison with other samples as shown in figure 5a. Higher magnification images revealed the presence of MWCNTs in the material (figure 5b, arrows). Similar to the two other samples, pores were connected to each other and formed a network of channels (figure 5c). However, the tubular pores extending perpendicularly to the surface of the sample were more irregular in this membrane than in pure PSU samples.

Figure 6 presents the pore size distribution in tested materials. For this evaluation, only pores bigger than $1 \mu \mathrm{m}$ were analysed, as small pores are not relevant either for the transport of nutrients or cell ingrowth. According to the literature, the smallest size of microspores, where ingrowth of the neighbouring bone tissue is possible, lies between 100 and $150 \mu \mathrm{m}$. Nevertheless, osteoconduction can occur occasionally when the pores size is only $50 \mu \mathrm{m}$ [34]. The optimal pore size, according to Chang et al [35], is $300 \mu \mathrm{m}$.

In general, the smallest pores could be observed for pure PSU membranes. About $60 \%$ of their pores had diameters in the range of $14.01-19 \mu \mathrm{m}$. For PSU+2\%SCF, this range contained less pores, only about $42 \%$. Simultaneously, for these membranes, more than twice the amount of pores with

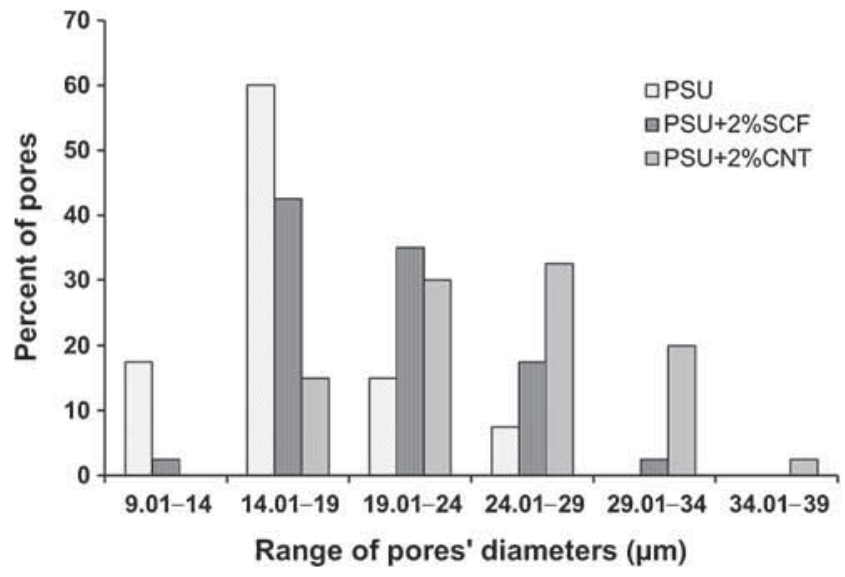

Figure 6. Pores distribution in PSU, PSU $+2 \% \mathrm{SCF}$ and PSU+ $2 \%$ CNT membranes.

diameters in the range of 19.01-24 $\mu \mathrm{m}$ was observed compared with pure PSU membranes. The addition of CNTs into polymer resulted in a slight increase of pore diameter; with nearly $30 \%$ of pores in the range of $24.01-29 \mu \mathrm{m}$. 
The increase in pore size of the PSU membrane modified with CNTs was also observed in the work of other authors [8]. Differences in pore size distribution can be caused by changes in the rheological properties of polymer solutions containing the carbon phase. In particular, this can be observed for the solution containing CNTs with a high specific surface area $\left(192 \mathrm{~m}^{2} \mathrm{~g}^{-1}\right)$. CNTs can reduce molecular mobility of polymer chains and also phase separation at different temperatures compared with the pure polymer [21,36,37]. Bose et al [36] reported that a concentration of $2 \mathrm{wt} \%$ MWCNTs clearly reduced macromolecular mobility of the blend components and induced phase separation at lower temperatures compared with the neat blends. In a similar study, the porosity growth for polymer membranes modified with CNTs was a result of the hydrophilic character of these nanomaterials [21]. This result might be explained by the fast exchange of solvent and non-solvent in the phase inversion process due to the hydrophilic properties of MWCNTs [21,38].

Although pore size values in obtained samples were smaller than reported elsewhere [34,35], the existence of these pores creates a hierarchical membrane structure and changes their roughness, which can positively influence adhesion and proliferation of bone cells. Moreover, the presence of hierarchical pores in the range of 5-70 $\mu \mathrm{m}$ allows for blood and interstitial flow in the space of biomaterials [39]. It is well known that bone tissue would not be viable without these fluid movements.

\subsection{Topography and surface roughness}

Bimodal pore distribution on the membrane surface allows different levels of roughness. Surface topography and roughness have a significant impact on cell adhesion to the surface of the material, especially in the case of bone cells [40]. In addition, several studies have proved that the existence of gradient roughness on the surface of membranes allows for better interaction between cells and the material $[41,42]$.

AFM and contact profilometry were used for analysis of surface topography and roughness of composite and pure PSU membranes. The use of AFM and profilometry allows obtaining surface roughness at the micro- and submicrometre scale. Figure 7a presents the surface topography of a representative pure PSU membrane using AFM. The maximum pore depth, calculated from a line profile analysis, is about $4 \mu \mathrm{m}$ (figure $7 \mathrm{~b}$ ). AFM analysis of the membrane surface between large pores revealed a morphology composed of smaller pores featuring much smaller diameters and depths (figure 7c).

The surface of the PSU+2\%SCF membrane showed a morphology similar to that of pure PSU membranes (figure 8a). Both micrometric pores and smaller pores were observed here. The depth of the micrometric pores $(2-3 \mu \mathrm{m})$ seemed to be smaller than the depth of this kind of pores in the PSU membrane (figure $8 b$ ).

The presence of $2 \% \mathrm{CNT}$ in PSU introduced only small changes in membrane morphology compared to membranes containing 2\% SCF. Although micrometric pores had a similar depth in the range of 2-3 $\mu \mathrm{m}$, the surface between these pores seemed to be less regular compared with PSU+2\%SCF (figure 9b). Such a relatively small change surface morphology can be driven by the presence of MWCNTs. Such surfaces were also more difficult to be measured because during scanning, the AFM tip displaced some surface material. The most probable cause of this effect was the interaction of the AFM tip with a single nanotube protruding on the surface (a)

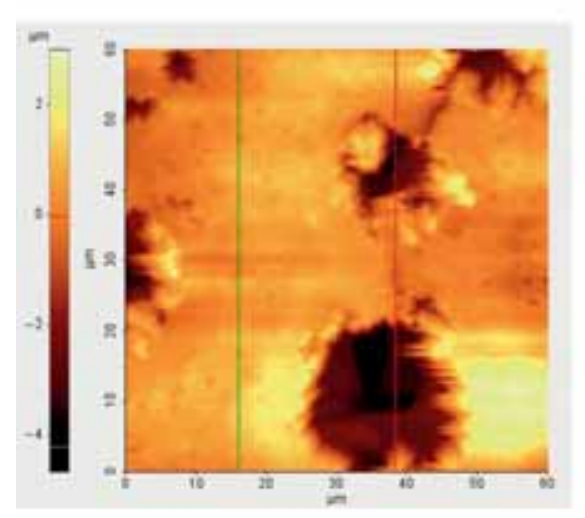

(b)

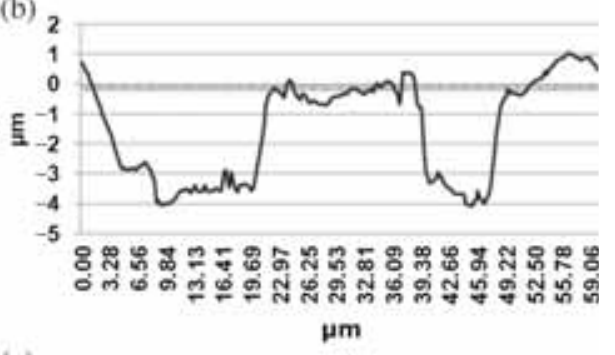

(c)

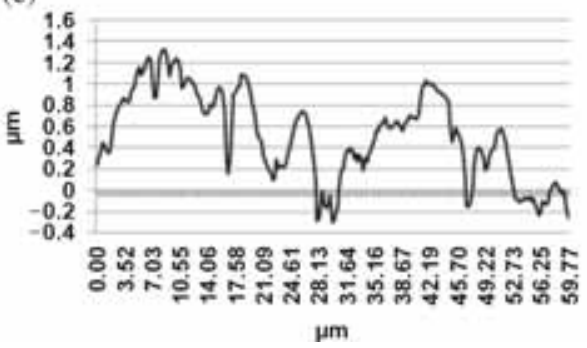

Figure 7. (a) Topography of pure PSU membrane surface $(60 \mu \mathrm{m} \times 60 \mu \mathrm{m})$, (b) line profile of the PSU surface with the large pore included (red line on the topography image) and (c) line profile of the PSU surface showing its morphology between the large pores (green line on the topography image). 
(a)

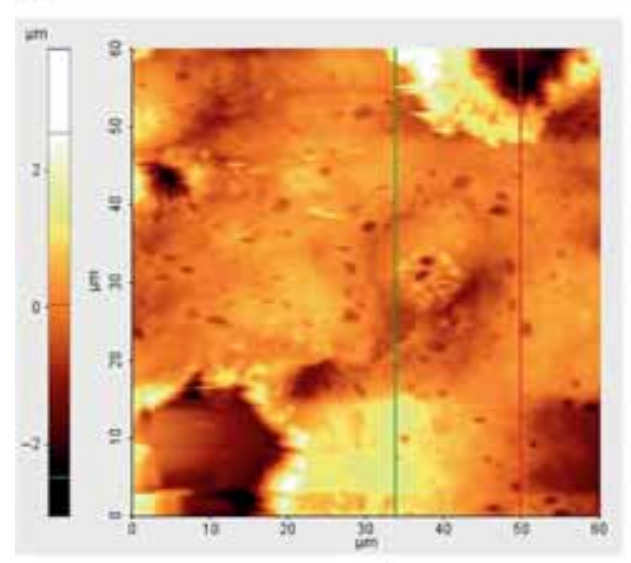

(b)

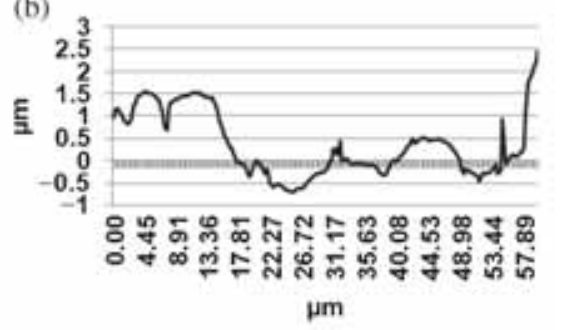

(c)

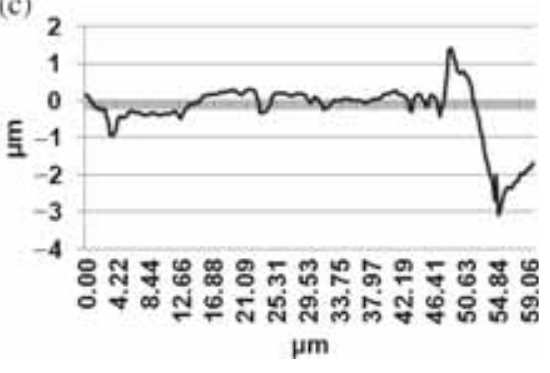

Figure 8. (a) Surface topography of PSU+2\%SCF membrane $(60 \mu \mathrm{m} \times 60 \mu \mathrm{m})$, (b) line profile of the PSU+2\%SCF surface with the large pore included (red line on the topography image) and (c) line profile of the PSU+2\%SCF surface between the big pores (green line on the topography image).

(a)

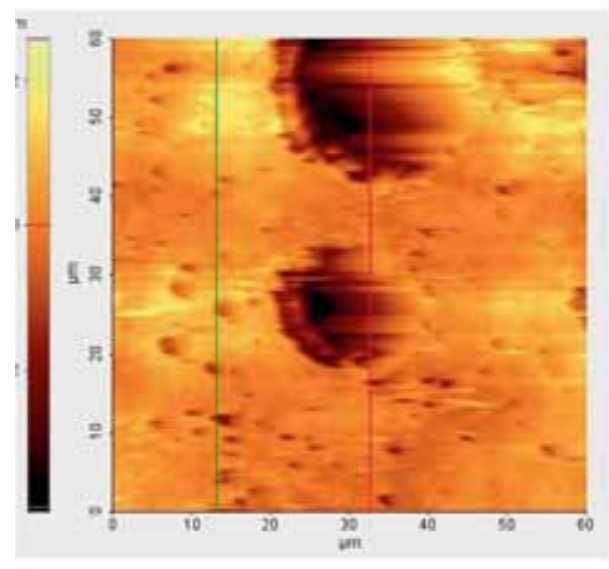

(b) 2

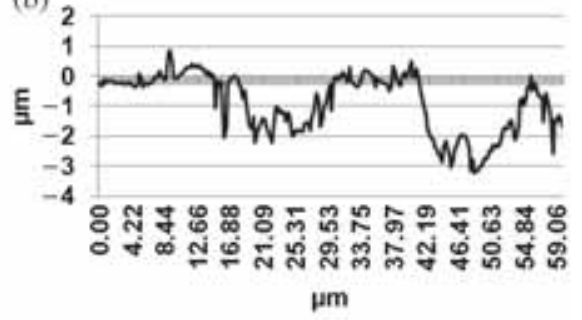

(c)

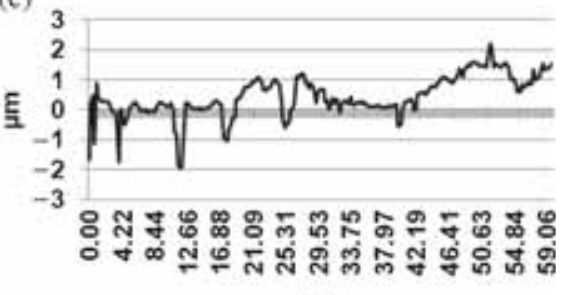

$\mu \mathrm{m}$

Figure 9. (a) Surface topography of PSU+2\%CNT membrane $(60 \mu \mathrm{m} \times 60 \mu \mathrm{m})$, (b) line profile of the PSU+2\%CNT surface with the large pore included (red line on the topography image) and (c) line profile of the PSU+2\%CNT surface between the large pores (green line on the topography image).

from the volume of material. This effect was visible as a horizontal line overlapping with the scan direction (figure 9a).

In order to evaluate the surface morphology in a quantitative way, two roughness parameters have been determined from the studied area, i.e., the average roughness value $\left(R_{\mathrm{a}}\right)$ and ten-point mean height $\left(R_{\mathrm{z}}\right)$. In the case of AFM, these parameters were calculated only for the sample surface between large pores, whereas in the case of the profilometry, they were calculated for the entire surface. A comparison of the surface roughness of all membranes is presented in figure $10 a$ and $b$.
AFM results analysis showed that the largest roughness values were obtained for PSU+2\%CNT membranes and the smallest for PSU+2\%SCF (figure 10a). It should be noted here that both parameters describing surface morphology showed similar trends for all samples. The large experimental errors (determined here as standard deviations) of the $R_{\mathrm{z}}$ parameter denote the magnitude of variations within a given sample type. Thus, a large error of a surface morphology value for a given PSU membrane also indicates a large variability in surface morphology. The addition of SCFs to PSU leads to decreased roughness, indicating that the surface 

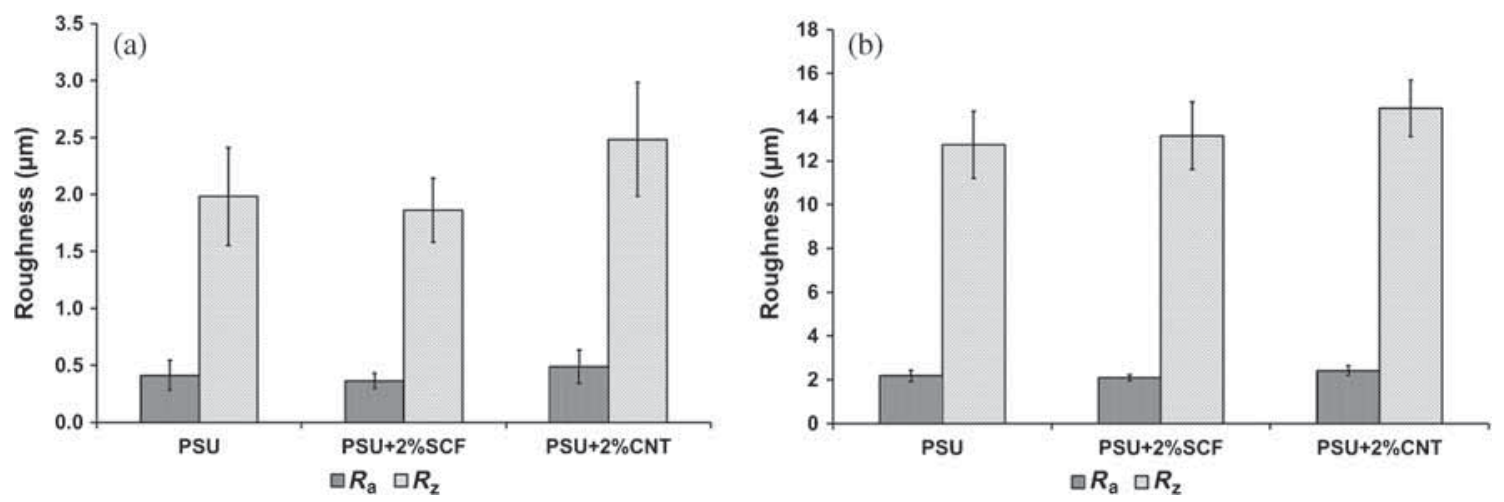

Figure 10. Comparison of roughness parameters determined for the studied membranes.

of PSU+2\%SCF is smoother and more homogenous than that of a pure PSU membrane. The results obtained using contact profilometry also show that highest roughness values were observed for samples containing CNTs (PSU+2\%CNT) (figure 10b). The surface roughness tendency of analysed samples using the profilometer and AFM was maintained, but in the case of profilometry, obtained values were about 5-6 times higher than those obtained from AFM. This is due to the areas of the analysed surface; for AFM, only surfaces without large pores were analysed, whereas in the case of the profilometer, the entire surface of the sample, containing also large pores, was investigated. Thus, the results confirm the existence of gradient roughness in the analysed samples.

The highest roughness both in terms of the parameter $R_{\mathrm{a}}$ and $R_{\mathrm{z}}$ was observed in membranes containing CNTs (PSU+2\%CNT), which is in accordance to the results obtained from the porosity analysis of these samples. In contrast to these results, Khalid et al [26] have observed a reduction of surface roughness of PSU membranes after addition of the CNTs (about 30\%) as compared with pure PSU membrane. They explained these results by the fact that a high CNTs' loading leads to an increase in viscosity of the casting solution and the exchange rate of solvent and non-solvent diffusion during immersion precipitation. However, these membranes have a much smaller number and size of pores than those shown in this publication, which results from different conditions of the phase inversion process proposed in these publications.

The differences in the roughness of PSU+2\% CNT membrane in micro- and submicrometre scale, compared with the pure PSU membrane described in this publication, are lower than in our previous article [20]. This is primarily due to the surface chemistry of MWCNTs. In the present publication CNTs have chemical groups such as $-\mathrm{OH}$ and $-\mathrm{COOH}$, which significantly impact on dispersion of these additives in DMF and PSU.

\subsection{Wettability and surface energy}

Microstructure, topography, roughness and the surface chemistry of biomaterials have a significant impact on the cell

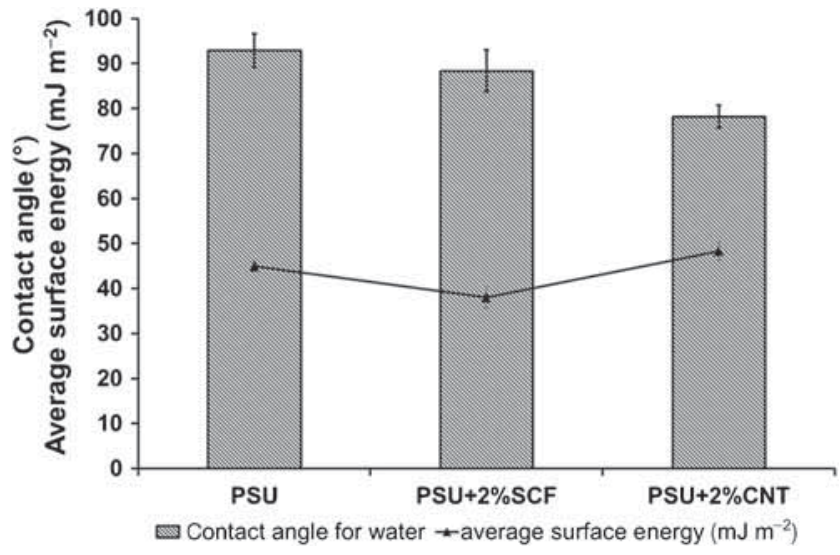

Figure 11. Contact angles and average surface energy of PSU, $\mathrm{PSU}+2 \% \mathrm{SCF}$ and $\mathrm{PSU}+2 \% \mathrm{CNT}$ membranes.

response. Among these parameters, wettability and surface energy play major roles in interaction with cells. The results of wettability and surface energy measurements are shown in figure 11. Average contact angles for PSU $+2 \% \mathrm{SCF}$ and PSU+2\%CNT membranes were lower than for pure PSU membrane. Thus, the addition of carbon material resulted in a decrease of the water contact angle, which is consistent with the data obtained by other researchers $[41,42]$. In the case of PSU $+2 \% \mathrm{CNT}$, the contact angle decreased by about $15 \%$. Lower contact angles of these membranes are connected with the presence of chemical groups such as $-\mathrm{OH}$ and $-\mathrm{COOH}$ on MWCNTs surfaces, which were created during the oxygenation process of these materials. This effect was confirmed in the work of de Lannoy et al [24] In our previous work [20] we did not observe any significant impact of CNTs on value of water contact angle as compared to the control sample (pure PSU), which results from the other type of nanotubes applied (non-functionalized) and a non-porous structure of the obtained nanocomposites.

The surface wettability of PSU+2\%SCF membranes was insignificantly higher compared to the pure PSU, but lower than for PSU containing MWCNTs. The main atomic constituent of the carbon fibre surface is carbon, but other elements, including oxygen, nitrogen, sulphur and silicon can also be present [43]. In a previous study, oxygen was present 
Table 1. Surface energy and its components' values in examined membranes.

\begin{tabular}{lccc}
\hline \multirow{2}{*}{ Material } & \multicolumn{3}{c}{ Surface energy $\left(\mathrm{mJ} \mathrm{m}^{-2}\right)$} \\
\cline { 2 - 4 }$\gamma_{\mathrm{p}}$ & $\gamma_{\mathrm{d}}$ & $\gamma$ \\
\hline PSU & $0.22 \pm 0.07$ & $44.71 \pm 1.14$ & $44.93 \pm 1.20$ \\
PSU+2\%SCF & $1.70 \pm 0.31$ & $36.33 \pm 1.94$ & $38.02 \pm 2.25$ \\
PSU+2\%CNT & $3.04 \pm 0.30$ & $45.25 \pm 1.68$ & $48.29 \pm 1.99$ \\
\hline
\end{tabular}

on the fibre surface in four forms: carbonyl, phenolic, ether or lactone structures [44]. The presence of these groups, especially the phenolic, which contains a hydroxyl group $(-\mathrm{OH})$, may have a negligible impact on the wettability of membrane surfaces modified with SCFs (PSU+2\%SCF). However, it should be noted that the concentration of surface functional groups on fibres that are not chemically oxidized is low, being about 0.045 to $0.075 \mathrm{~mol} \mathrm{~g}^{-1}$ [45].

Table 1 presents the values of the polar component $\left(\gamma_{p}\right)$ and the dispersive component $\left(\gamma_{\mathrm{d}}\right)$ of the surface energy and also an average surface energy $(\gamma)$ of all investigated materials. The values of average surface energy are also presented in figure 11.

The surface energy of pure PSU practically consists of only one dispersive component. This is connected with the fact that PSU has a hydrophobic nature. Modification of the polymer with carbon additives changes the proportions between the dispersive and polar components: the polar component grows, while the dispersive component decreases. The significant increase of the polar component of surface energy for PSU $+2 \% \mathrm{CNT}$, in comparison with the rest of the membranes, indicates and confirms a more hydrophilic character of this membrane type. The average surface energy for PSU+2\%CNT was higher than for pure PSU and PSU+2\%SCF. Moreover, the growth of surface energy was interrelated with the decrease of the contact angle (figure 11). These parameters have a great meaning in bone applications of materials. Researchers suggest that hydrophilic surfaces with higher surface energy promote osteoblasts growth and mineralization [46].

\subsection{Mechanical properties of composites}

Membranes used in the GTR technique should have appropriate physicochemical, biological and mechanical properties in order to play a decisive role in tissue healing. The ability to control the mechanical properties of biomaterials allows researchers to tune membranes, depending on the intended application. For example, flexible membranes are needed for mechanical conditioning of cells in compression; however, membranes need to be stiff enough to fill a loadbearing defect and to be handled during surgery [47]. PSUs are thermoplastic polymers with very good mechanical properties and therefore ideally suited for bone cells regeneration. The main aim in the addition of different kinds of carbon additives to PSU was to verify to what extent they affect tensile strength and the Young's modulus of the composites.
Figure 12 displays tensile strength $\left(R_{\mathrm{m}}\right)$ and Young's modulus $(E)$ of the studied membranes. The highest recorded value of tensile strength was featured by the PSU $+2 \% \mathrm{SCF}$ membrane. As expected, the parallel alignment of the fibrous reinforcement phase (figure 13) had a significant influence on the mechanical parameters of the final composite. This situation specifically refers to long-fibre composites; however, in this case, where short fibres exist, reinforcement was also observed (figure 13).

Tensile strength for PSU+2\%SCF was approximately 14 and 21\% higher compared with pure PSU and $\mathrm{PSU}+2 \% \mathrm{CNT}$, respectively. For PSU+2\%CNT membranes, tensile strength was similar and even insignificantly lower compared to pure PSU. The lack of reinforcement for this sample can be connected to the tendency of CNTs to agglomerate. Agglomerates of CNTs, in contrast with the pure polymer, can be considered as a defect, which can cause lack of reinforcement or even loss of tensile strength. The adverse effect of CNTs agglomerates on the mechanical properties of PSU has been also observed by other researchers $[27,48]$.

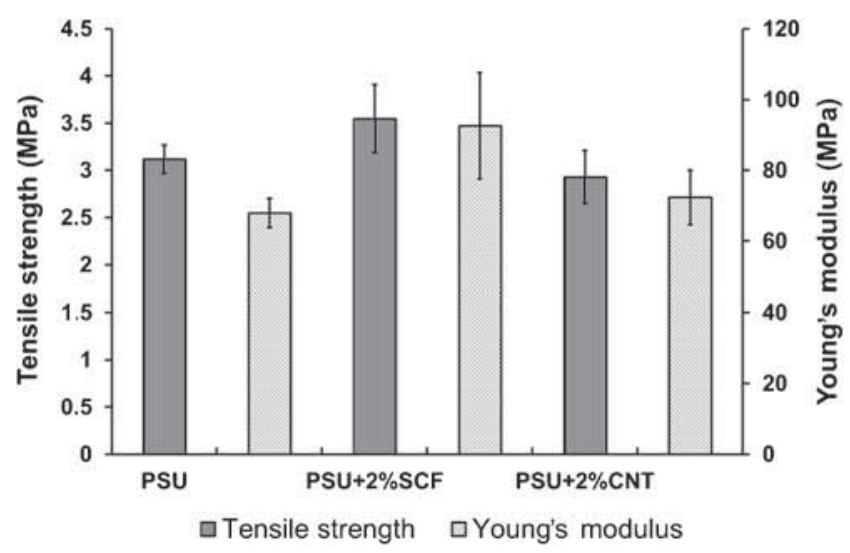

Figure 12. Tensile strength and Young's modulus of examined membranes.

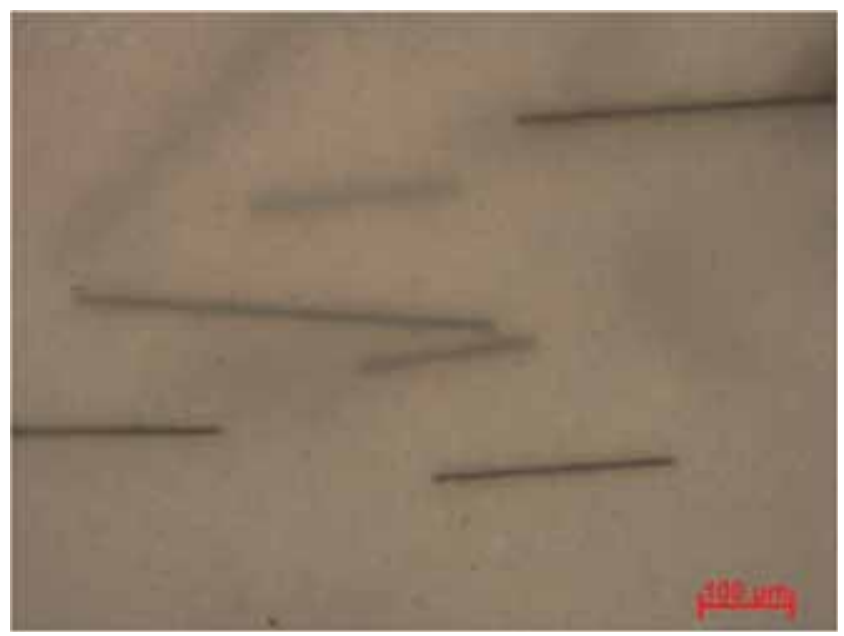

Figure 13. Microphotography of PSU $+2 \% \mathrm{SCF}$ membrane, showing parallel asignment of short fibres in the polymer. Magnification 10×. 
Moreover, a more likely reason of insignificant reduction of tensile strength could be connected to higher pore amount compared with the rest of the samples. Analysing the results of our previous studies of nanocomposites based on PSU containing $2 \%$ of CNTs, the growth of the mechanical properties in the range of $20-30 \%$ compared with pure PSU was observed despite a significant amount of agglomerates of CNTs and lack of chemical groups of their surface [20]. These results may confirm more substantial impact of the porosity on the mechanical properties of PSU $+2 \% \mathrm{CNT}$ than the presence of CNTs agglomerates. However, the addition of CNTs (PSU+2\%CNT) did not significantly deteriorate mechanical properties of the membrane for the regeneration of bone tissue.

Figure 12 also presents values of the Young's modulus for all samples. The highest value of Young's modulus $(E)$ was measured for PSU+2\%SCF membranes. Young's modulus for this sample increased over $36 \%$ and almost $28 \%$ compared with pure PSU and PSU+2\%CNT samples, respectively. The increase in the Young's modulus for PSU+2\%SCF membranes can be connected to the directional orientation of the fibres along the axis of the breaking force. Moreover, it is an indirect evidence of a good interaction at the interface between fibres and matrix. The elastic modulus measured for PSU+2\%CNT membranes was similar to that measured for PSU membranes.

\subsection{Preliminary in vitro study}

PSU membranes without carbon additives and those modified with SCFs and MWCNTs were cultured with MG-63 osteoblast-like cells for 3 and 7 days. Analysis of cell viability after 7 days revealed significant differences between the samples (figure 14b).

Cell viability after 7 days of culture in contact with pure PSU and PSU+2\%SCF membranes was higher compared with the positive control (polystyrene culture dish), whereas cell viability for PSU+2\%CNT was insignificantly lower compared to the control sample. Addition of SCFs to PSU significantly increased cell viability (30\%) compared with pure PSU samples. This effect was not observed with $2 \mathrm{wt} \%$ of MWCNT; here, cell viability decreased about $20 \%$ compared with the PSU sample. PSU is a biostable polymer; therefore, the release of nanotubes into the culture medium does not occur. For this sample, CNTs were observed both on the surface of membrane as well as inside the pores, anchored in the polymer, in contrast with SCFs, which were completely immersed in the PSU. In this situation, factors such as length, concentration, tendency to agglomerate and transport of nanotubes through the cell membrane directly to individual organelles inside the cell should not be taken into consideration during the interpretation of these results. These factors are usually taken into account when assessing the toxicity of CNTs [49-54]. Moreover, the purity of these samples was rather high (the concentration of metal catalyst $(\mathrm{Fe})$ was $0.06 \mathrm{wt} \%$ ). Surface chemistry (type of functional groups) is another factor frequently mentioned in the literature; it may affect the biocompatibility of nanotubes $[51,55]$. In this study, the factor associated with nanotubes may be the most important one when interpreting the results of biological research. The carboxyl and hydroxyl groups are probably the most popular functional groups on CNTs. The presence of these groups changes the hydrophilicity of CNT, which allows this material to be well dispersed in aqueous solutions, including culture media, as well as in other polar solvents, and enables further functionalization of nanotubes $[13,21,56]$. However, studies often indicate the positive influence of these functional groups on biocompatibility, particularly in contact with bone cells [57-59]. Surface development of CNTs is another factor that could have an influence on the cells response. High surface development of CNTs causes an increase in the amount of active sites ready for adsorption and/or binding to other molecules or substances. Thus, the combination of high surface development of nanotubes and the presence of functional groups on the surface may cause binding and adsorption of nutrients from the culture medium, thereby depleting these components. This may have an indirect effect on the number of cells adherent to the substrate containing the nanotubes (figure 14a) and, consequently, on cell viability (figure 14b). Moreover, the decrease of MG-63
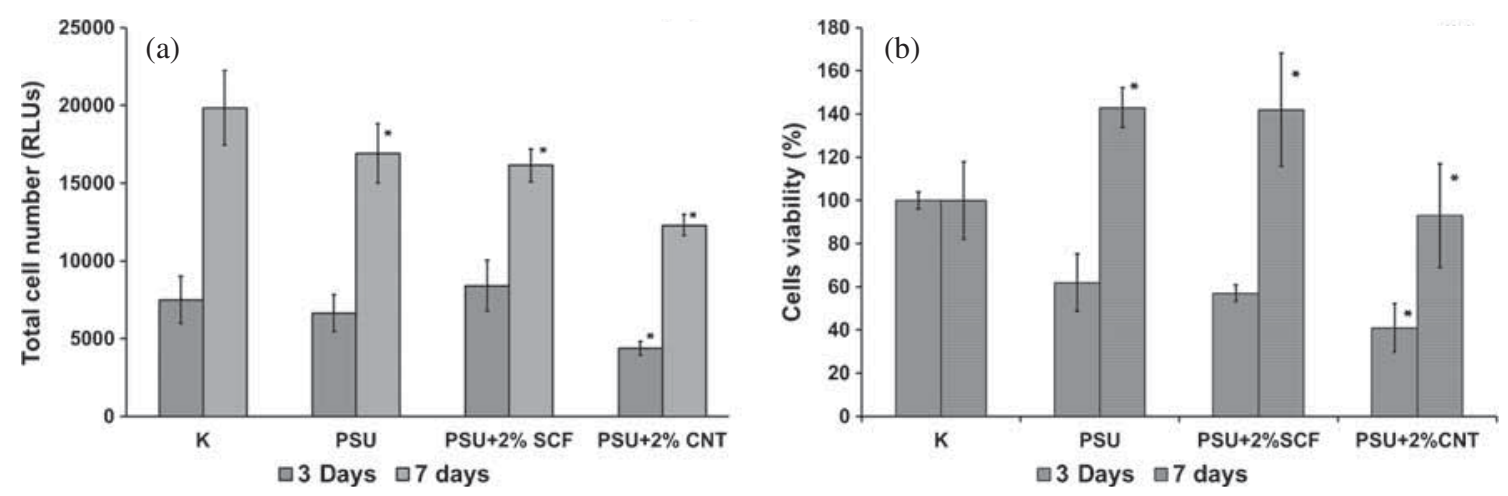

Figure 14. (a) MG-63 proliferation and (b) cells viability in contact with membranes after 3 and 7 days of culture. The data are expressed as mean \pm SD from 5 to 8 measurements, Student's $t$-test. ${ }^{*}$ Statistically significant difference compared with controls (cells cultured without CNTs $=$ tissue culture in polystyrene) $(p<0.05)$. 
viability in contact with PSU $+2 \%$ CNT can be connected to the porosity of this membrane. For this sample, pores sizes were larger compared with the rest of the samples (figures 5 and 6). The presence of pores with diameters in the range of 24-40 $\mu \mathrm{m}$ for PSU+2\%CNT membrane allows the penetration of single cells into the materials (the MG63 cell size is about $20-40 \mu \mathrm{m}$ ) and partially reduces their growth. Moreover, impregnability of membranes containing 2 wt $\%$ of MWCNTs increased over $40 \%$ compared with pure PSU membranes (data not shown). As a result, nutrients are retained in the pores of the composites and cannot be accessed by the cells. The measurement of cells viability may also be affected by an error resulting from the difficult access of tetrazolium salt to the interior of the material and the consequent lack of reduction of this salt to formazan.

Compared with a cultivation period of 3 days, cell viability increased after a period of 7 days in all samples. A lack of inhibition of cell proliferation and the continued growth of cell viability vs. time may indicate that all analysed membranes are biocompatible in contact with bone cells. In our previous work we also observed acceleration of cells proliferation from one day to another in contact with PSU/MWCNTs, although the method of composites preparation and surface chemistry of nanotubes were different [20]. Biological studies carried out in the earlier work [20] demonstrate that CNTs presence in the PSU did not significantly stimulate production of markers responsible for cell immune activation in MG-63 cells. These results showed relatively good support for the adhesion and growth of osteogenic cells.

\section{Conclusions}

Three types of PSU-based membranes, obtained using the phase inversion method, were analysed in terms of physicochemical and biological properties as potential materials for bone cells growth. Two of them were modified using carbon fibrous additives, i.e., MWCNTs and SCFs. The presence of different kinds of carbon fibrous materials in the PSU matrix can influence the physicochemical and biological properties of the obtained composite membranes in different ways. These changes can be observed in microstructure, topography, wettability, surface energy and mechanical properties of the obtained composite samples. The differences in dimension, surface chemistry and surface energy of SCFs and CNTs have a crucial impact on physicochemical properties of the composites, and simultaneously on the MG-63 cells response.

The presence of hydrophilic groups, especially on the surface of CNTs, can change the mechanism of solvent exchange on non-solvent during the phase inversion, which could imply a different porosity composite containing CNTs when compared with pure PSU membrane. Also, wettability of composite membranes was lower in comparison with membranes without carbon additives. Directionally arranged SCFs in PSU membranes had a positive impact on the mechanical properties of the composite samples. A lack of strengthening effect and even a decrease of the mechanical properties were observed for membranes modified with 2 wt $\%$ of MWCNTs; this could be due to the agglomeration of the carbon nanostructures within the polymer matrix. The preliminary in vitro study indicated that all membranes were biocompatible in contact with MG-63 cells, but the mechanism of interaction between cells and composites was different and strongly depended on the porosity of membranes, the presence of carbon additives on the membrane surface and the surface development of carbon fillers and their chemistry.

\section{Acknowledgements}

The study was financed from the statute funds of AGHUST, Faculty of Materials Engineering and Ceramics, Project no.11.11.160.616. M T acknowledges the financial support from the U.S. Air Force Office of Scientific Research, through MURI grant FA9550-12-1-0035.

\section{References}

[1] Baker R 2004 Membrane technology and applications (Chichester, West Sussex: John Wiley \& Sons Ltd)

[2] Krause B, Storr M, Ertl T, Buck R, Hildwein H, Deppisch R and Göhl H 2003 Chem. Ing. Tech. 751725

[3] Stamatialis D F, Papenburg B J, Gironés M, Saiful S, Bettahalli S N, Schmitmeier S and Wessling M $2008 \mathrm{~J}$. Membr. Sci. $\mathbf{3 0 8} 1$

[4] Retzepi M and Donos N 2010 Clin. Oral Implants Res. 21567

[5] Milella E, Ramires P A, Brescia E, La Sala G, Di Paola L and Bruno V 2001 J. Biomed. Mater. Res. Part A 58427

[6] Wang H-L, Miyauchi M and Takata T 2002 J. Periodontal Res. 37340

[7] Strietzel F P, Khongkhunthian P, Khattiya R, Patchanee P and Reichart P A 2006 J. Biomed. Mater. Res. Part B 7835

[8] Nechifor G, Voicu S, Nechifor A and Garea S 2009 Desalination 241342

[9] Blazewicz M 2001 Eur. Cells Mater. 221

[10] Li C S, Vannabouathong C, Sprague S and Bhandari M 2015 Clin. Med. Insights Arthritis Musculoskelet. Disord. 833

[11] Rajzer I, Menaszek E, Bacakova L, Rom M and Blazewicz M 2010 J. Mater. Sci. Mater. Med. 212611

[12] Rajzer I, Rom M and Blazewicz M 2010 Fibers Polym. 11615

[13] Harrison B S and Atala A 2007 Biomaterials 28344

[14] Tran P A, Zhang L and Webster T J 2009 Adv. Drug Deliv. Rev. 611097

[15] Chlopek J, Czajkowska B, Szaraniec B, Frackowiak E, Szostak K and Beguin F 2006 Carbon 441106

[16] Deligianni D D 2014 Cell Adhes. Migrat. 8558

[17] Newman P, Minett A, Ellis-Behnke R and Zreiqat H 2013 Nanomedicine $\mathbf{9} 1139$

[18] Pan L, Pei X, He R, Wan Q and Wang J 2012 Colloids Surf. B Biointerfaces 93226

[19] Sitharaman B, Shi X, Walboomers X F, Liao H, Cuijpers V, Wilson L J, Mikos A G and Jansen J A 2008 Bone 43362 
[20] Stankova L, Fraczek-Szczypta A, Blazewicz M, Filova E, Blazewicz S, Lisa V and Bacakova L 2014 Carbon 67578

[21] Choi J-H, Jegal J and Kim W-N 2006 J. Membr. Sci. 284 406

[22] Geise G M, Lee H-S, Miller D J, Freeman B D, McGrath J E and Paul D R 2010 J. Polym. Sci. Part B: Polym. Phys. 48 1685

[23] Yuenyao C, Tirawanichakul Y and Chittrakarn T 2015 J. Appl. Polym. Sci. 132

[24] De Lannoy C-F, Soyer E and Wiesner M R 2013 J. Membr. Sci. 447395

[25] Fernandes G, Pinto J and Nobrega R $2001 \mathrm{~J}$. Appl. Polym. Sci. 823036

[26] Khalid A, Al-Juhani A A, Al-Hamouz O C, Laoui T, Khan Z and Atieh M A 2015 Desalination 367134

[27] Maphutha S, Moothi K, Meyyappan M and Iyuke S E 2013 Sci. Rep. 3

[28] Sedláková Z, Clarizia G, Bernardo P, Jansen J C, Slobodian P, Svoboda P, Kárászová M, Friess K and Izak P 2014 Membranes 420

[29] Carrero-Sanchez J, Elias A, Mancilla R, Arrellin G, Terrones H, Laclette J and Terrones M 2006 Nano Lett. 61609

[30] Simone S, Figoli A, Criscuoli A, Carnevale M, Rosselli A and Drioli E 2010 J. Membr. Sci. 364219

[31] Fraczek A and Blazewicz S 2009 J. Phys. Conf. Ser. 146 012005

[32] Fraczek-Szczypta A and Blazewicz S 2011 J. Mater. Sci. 46 5680

[33] Owens D K and Wendt R C 1969 J. Appl. Polym. Sci. 131741

[34] Sopyan I, Mel M, Ramesh S and Khalid K 2007 Sci. Technol. Adv. Mater. 8116

[35] Chang B S, Lee C K, Hong K S, Youn H J, Ryu H S, Chung S S and Park K W 2000 Biomaterials 211291

[36] Bose S, Cardinaels R, Özdilek C, Leys J, Seo J W, Wübbenhorst M and Moldenaers P 2014 Eur. Polym. J. 53253

[37] Nobile M 2011 Polymer-carbon nanotube composites: preparation, properties and applications (Sawston, Cambridge: Woodhead Publishing) 15428

[38] Wienk I, Boom R, Beerlage M, Bulte A, Smolders C and Strathmann H 1996 J. Membr. Sci. 113361

[39] Cowin S C and Cardoso L 2015 J. Biomech. 48842

[40] Imade S, Mori R, Uchio Y and Furuya S 2009 J. Orthop. Sci. 14652

[41] Faia-Torres A B, Charnley M, Goren T, Guimond-Lischer S, Rottmar M, Maniura-Weber K, Spencer N D, Reis R L, Textor M and Neves N M 2015 Acta Biomater. 2864
[42] Gittens R A, McLachlan T, Olivares-Navarrete R, Cai Y, Berner S, Tannenbaum R, Schwartz Z, Sandhage K H and Boyan B D 2011 Biomaterials 323395

[43] Hopfgarten F 1978 Fibre Sci. Technol. 1167

[44] Boehm H-P, Diehl E, Heck W and Sappok R 1964 Angew. Chem. Int. Ed. Engl. 3669

[45] Pampuch R 1986 Włókna weglowe (Wydawnictwo AGH: Krakow)

[46] Lim J Y, Shaughnessy M C, Zhou Z, Noh H, Vogler E A and Donahue H J 2008 Biomaterials 291776

[47] Owen R, Sherborne C, Paterson T, Green N H, Reilly G C and Claeyssens F 2016 J. Mech. Behav. Biomed. 54159

[48] Brunet L, Lyon D, Zodrow K, Rouch J-C, Caussat B, Serp P, Remigy J-C, Wiesner M and Alvarez P 2008 Environ. Eng. Sci. 25565

[49] Aillon K L, Xie Y, El-Gendy N, Berkland C J and Forrest M L 2009 Adv. Drug Deliv. Rev. 61457

[50] Fraczek-Szczypta A, Menaszek E and Blazewicz S $2011 \mathrm{~J}$. Nanomater. 5

[51] Fraczek-Szczypta A, Menaszek E, Blazewicz S, Adu J, Shevchenko R, Syeda T B, Misra A and Alavijeh M 2015 Mater. Sci. Eng. C $\mathbf{4 6} 218$

[52] Kagan V, Tyurina Y, Tyurin V, Konduru N, Potapovich A, Osipov A, Kisin E, Schwegler-Berry D, Mercer R, Castranova V and Shvedova A 2006 Toxicol. Lett. 16588

[53] Sato Y, Yokoyama A, Shibata K, Akimoto Y, Ogino S-I, Nodasaka Y, Kohgo T, Tamura K, Akasaka T, Uo M, Motomiya K, Jeyadevan B, Ishiguro M, Hatakeyama R, Watari F and Tohji K 2005 Mol. Biosyst. 1176

[54] Wick P, Manser P, Limbach L K, Dettlaff-Weglikowska U, Krumeich F, Roth S, Stark W J and Bruinink A 2007 Toxicol. Lett. 168121

[55] Coccini T, Roda E, Sarigiannis D, Mustarelli P, Quartarone E, Profumo A and Manzo L 2010 Toxicology 26941

[56] Saito N, Usui Y, Aoki K, Narita N, Shimizu M, Ogiwara N, Nakamura K, Ishigaki N, Kato H, Taruta S and Endo M 2008 Curr. Med. Chem. 15523

[57] Flores-Cedillo M, Alvarado-Estrada K, Pozos-Guillén A, Murgu-Ibarra J, Vidal M, Cervantes-Uc J, Rosales-Ibáñez R and Cauich-Rodríguez J 2016 J. Mater. Sci.: Mater. Med. 271

[58] Saffar K P, Rouhi G and JamilPour N 2009 Carbon nanotubes in bone tissue engineering (Croatia: INTECH Open Access Publisher)

[59] Saito N, Haniu H, Usui Y, Aoki K, Hara K, Takanashi S, Shimizu M, Narita N, Okamoto M, Kobayashi S, Nomura H, Kato H, Nishimura N, Taruta S and Endo M 2014 Chem. Rev. 1146040 